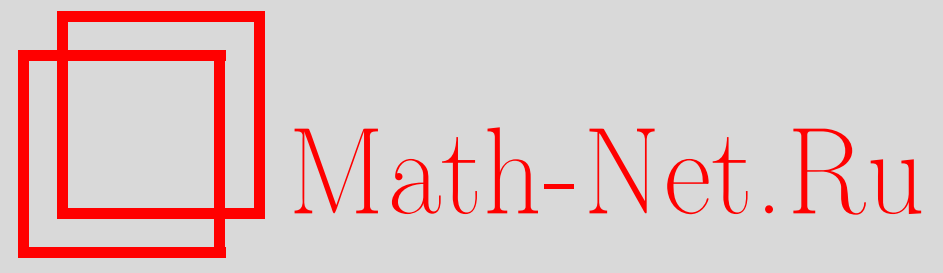

Г. П. Чистяков, Новое асимптотическое разложение и асимптотически наилучшие постоянные в теореме Ляпунова. II, Теория вероятн. и ее примен., 2001, том 46, выпуск 3, 573-579

DOI: https://doi.org/10.4213/tvp3905

Использование Общероссийского математического портала Math-Net.Ru подразумевает, что вы прочитали и согласны с пользовательским соглашением http://www . mathnet.ru/rus/agreement

Параметры загрузки:

IP : 35.173 .219 .149

26 апреля 2023 г., $10: 16: 42$

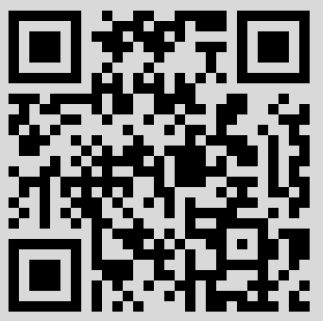


(C) $2001 \mathrm{r}$.

чИСТЯКОВ Г. П.*

\title{
НОВОЕ АСИМПТОТИЧЕСКОЕ РАЗЛОЖЕНИЕ И АСИМПТОТИЧЕСКИ НАИЛУЧШИЕ ПОСТОЯННЫЕ В ТЕОРЕМЕ ЛЯПУНОВА. ІІ ${ }^{1)}$
}

\begin{abstract}
В центральной предельной теореме Ляпунова получено новое асимптотическое разложение для функций распределения центрированных и нормированных сумм независимых случайных величин, необязательно одинаково распределенных. Полученное разложение используется для нахождения асимптотически наилучших постоянных в неравенстве Берри-Эссеена. Тем самым решена задача, поставленная Колмогоровым и Золотаревым.
\end{abstract}

Ключевые слова и фразы: центральная предельная теорема, теорема Ляпунова, оценки Берри-Эссеена, асимптотическое разложение, характеристические функции.

\section{4. Доказательство результатов об асимптотически наилучших постоянных}

Д ок а 3 а тел л с т в о те о р е мы 2.2. Мы выведем теорему 2.2 из теоремы 2.1. В §§ 4 и 5 будем использовать обозначения, введенные в §§ 1 и 2. Без потери общности предположим, что отношение Ляпунова $L \leqslant c$, где $c$ так мало, как потребуется в рассуждениях ниже.

Вначале докажем теорему в случае, когда $M_{f}>L^{2}, S_{*}=\varnothing$, и в случае, когда $M_{f} \leqslant L^{2}$. Для того, чтобы использовать вторую часть теоремы 2.1 , необходимо оценить величину $\alpha(x)$. По формуле Тейлора, $\alpha(x)$ можно записать в виде

$$
\alpha(x)=-\frac{1}{6 s^{3}} \sum_{j=1}^{n} \int_{-\infty}^{\infty} \Phi^{(3)}\left(x-\frac{u_{0}}{s}\right) u^{3} d F_{j}(u),
$$

где $u_{0}=u_{0}(u)$ принимает вещественные значения и $\left|u_{0}\right|<|u|$. Легко видеть, что

$$
\left|\Phi^{(3)}\left(x-\frac{u_{0}}{s}\right)\right| \leqslant \frac{1}{\sqrt{2 \pi}}
$$

и

$$
\left|\Phi^{(3)}\left(x-\frac{u_{0}}{s}\right)-\Phi^{(3)}(x)\right| \leqslant \sup _{v \in \mathbf{R}^{1}}\left|\Phi^{(4)}\left(x-\frac{v}{s}\right)\right| \frac{|u|}{s} \leqslant \frac{|u|}{s} .
$$

В силу (4.1) и (4.2), справедлива оценка

$$
|\alpha(x)| \leqslant \frac{1}{6 \sqrt{2 \pi}} \frac{1}{s^{3}} \sum_{j=1}^{n} \int_{-\infty}^{\infty}|u|^{3} d F_{j}(u)=\frac{1}{6 \sqrt{2 \pi}} L,
$$

из которой, учитывая вторую часть теоремы 2.1, следует, что

$$
\rho\left(F * \Phi_{\varepsilon}, \Phi\right) \leqslant \frac{1}{6 \sqrt{2 \pi}} L+c L^{1+c_{1}}|\ln L|^{7 / 6} .
$$

Chistyakov G. P. A new asymptotic expansion and asymptotically best constants in Lyapunov's theorem. II.

(C) Перевод на русский язык, Научное издательство «ТВП», 2001.

* Физико-технический институт низких температур им. Б.И. Веркина НАН Украины, пр. Ленина, 47, Харьков, 61164 Украина; e-mail: chistyakov@ilt.kharkov.ua

1) Часть I статьи опубликована в журнале «Теория вероятностей и ее применения», 2001, т. 46, в. 2, с. 326-344. Работа выполнена при поддержке SFB 343, Билефельд, Германия. 
Очевидно, что $\rho\left(\Phi * \Phi_{\varepsilon}, \Phi\right) \leqslant c L^{2}$. Теперь, применяя лемму 3.1 к плотностям $P_{1}$, $P_{2}$ и $H$ с функциями распределения $F(x), \Phi(x)$ и $\Phi_{\varepsilon}(x)$ соответственно, и полагая $\tau=10 \varepsilon \sqrt{|\ln \varepsilon|}$, приходим к оценке

$$
\rho(F, \Phi) \leqslant \frac{1}{6 \sqrt{2 \pi}} L+c L^{1+c_{1}}|\ln L|^{7 / 6}
$$

что доказывает теорему в рассматриваемом случае.

Теперь положим $M_{f}>L^{2}$ и $S_{*} \neq \varnothing$. Положим $\delta=L^{-5 / 12} h / s$ и перепишем формулу (4.1) в виде

$$
\begin{aligned}
\alpha(x)= & -\frac{1}{6 s^{3}} \sum_{j=1}^{n} \int_{|u| \leqslant \delta s} \Phi^{(3)}\left(x-\frac{u_{0}}{s}\right) u^{3} d F_{j}(u) \\
& -\frac{1}{6 s^{3}} \sum_{j=1}^{n} \int_{|u|>\delta s} \Phi^{(3)}\left(x-\frac{u_{0}}{s}\right) u^{3} d F_{j}(u)=\alpha_{1}(x)+\alpha_{2}(x) .
\end{aligned}
$$

В силу (4.2), член $\alpha_{2}(x)$ оценивается следующим образом:

$$
\left|\alpha_{2}(x)\right| \leqslant \frac{1}{6 \sqrt{2 \pi} s^{3}} \sum_{j=1}^{n} \int_{|u|>\delta s}|u|^{3} d F_{j}(u) .
$$

Используя оценку (4.3), перепишем член $\alpha_{1}(x)$ в виде

$$
\alpha_{1}(x)=\frac{1}{6 \sqrt{2 \pi}}\left(1-x^{2}\right) e^{-x^{2} / 2} \frac{1}{s^{3}} \sum_{j=1}^{n} \int_{|u| \leqslant \delta s} u^{3} d F_{j}(u)+\Delta(x)=\alpha_{1,1}(x)+\Delta(x),
$$

где

$$
|\Delta(x)| \leqslant c \frac{\delta}{s^{3}} \sum_{j=1}^{n} \int_{|u| \leqslant \delta s}|u|^{3} d F_{j}(u) \leqslant c \delta L .
$$

В силу $(2.9), \delta \leqslant c L^{7 / 12}$ и, наконец,

$$
|\Delta(x)| \leqslant c L^{19 / 12} .
$$

Величина $\alpha_{1,1}(x)$, очевидно, оценивается следуюшим образом:

$$
\left|\alpha_{1,1}(x)\right| \leqslant \frac{1}{6 \sqrt{2 \pi}} \frac{1}{s^{3}} \sum_{j=1}^{n}\left|\int_{|u| \leqslant \delta s} u^{3} d F_{j}(u)\right| .
$$

Теперь заметим, что модуль третьего члена в правой части (2.8) не превосходит

$$
\frac{h}{2 \sqrt{2 \pi} s} e^{-x^{2} / 2}
$$

Введем

$$
\gamma_{J_{*}}=\frac{1}{6 \sqrt{2 \pi}} \frac{1}{s^{3}} \sum_{j \in J_{*}}\left|\int_{|u| \leqslant \delta s} u^{3} d F_{j}(u)\right|+\frac{h}{2 \sqrt{2 \pi} s}
$$

где $J_{*}-$ множество индексов из теоремы 2.1. Используя (4.5)-(4.8), из представления (2.8) получим неравенство

$$
\begin{aligned}
\rho\left(F * \Phi_{\varepsilon}, \Phi\right) \leqslant & \gamma_{J_{*}}+\frac{1}{6 \sqrt{2 \pi} s^{3}} \sum_{j \in J_{*}} \int_{|u|>\delta s}|u|^{3} d F_{j}(u) \\
& +\frac{1}{6 \sqrt{2 \pi} s^{3}} \sum_{j \notin J_{*}} \beta_{j}+c L^{1+c_{1}}|\ln L|^{7 / 6}
\end{aligned}
$$


Введем случайные величины $Y_{j}=X_{j} / h$ с функциями распределения $G_{j}(x)=$ $F_{j}(h x)$ и харахтеристическими функциями $g_{j}(t)=f_{j}(t / h)$ соответственно. Легко видеть, что

$$
\mathbf{E} Y_{j}=0, \quad \mathbf{D} Y_{j}=\frac{\sigma_{j}^{2}}{h^{2}}, \quad \mathbf{E}\left|Y_{j}\right|^{3}=\frac{\beta_{j}}{h^{3}} .
$$

Покажем теперь, что случайные величины $Y_{j}, j \in J_{*}$, удовлетворяют условиям леммы 3.5 с $\eta=L^{2-13 c_{1}}$ и $K=6 c_{1}$. Для этого воспользуемся $(2.11)$ и оценкой $h \leqslant 10^{3} L s$, которая слабее, чем (2.9). Используя эти оценки, мы видим, что

$$
\left|g_{j}(2 \pi)\right|=\left|f_{j}\left(\frac{2 \pi}{h}\right)\right| \geqslant 1+c \frac{\ln L}{L^{12 c_{1}}} \frac{\sigma_{j}^{2}}{s^{2}}=1+c \frac{\ln L}{L^{12 c_{1}}}\left(\frac{h}{s}\right)^{2} \mathbf{D} Y_{j} \geqslant 1-L^{2-13 c_{1}} \mathbf{D} Y_{j},
$$

значит условие (3.5) выполняется. Положим $T=s^{2} / B$. В силу (2.4),

$$
\sigma_{j}^{2} T^{2}=\sigma_{j}^{2} \frac{s^{4}}{B^{2}} \leqslant \sigma_{j}^{2} L^{-16 c_{1}} \frac{\sigma_{j}^{4}}{\beta_{j}^{2}} \leqslant L^{-16 c_{1}},
$$

и поэтому, учитывая неравенство $h T \geqslant L^{c_{1}}$, которое следует из определения параметра $h$ (см. (2.7)), получаем $\mathrm{D} Y_{j} \leqslant L^{-16 c_{1}}(h T)^{-2} \leqslant L^{-18 c_{1}}$, так что условие (3.3) также выполняется. Вновь используя (2.4), получаем

$$
\mathbf{E}\left|Y_{j}\right|^{3}=\frac{\beta_{j}}{B} \frac{B}{h^{3}} \leqslant L^{-8 c_{1}} \frac{\sigma_{j}^{2}}{s^{2}} \frac{B}{h^{3}}=L^{-8 c_{1}}(h T)^{-1} \mathbf{D} Y_{j} \leqslant L^{-9 c_{1}} \mathrm{D} Y_{j},
$$

т.е. условие (3.4) выполняется. Таким образом, все условия леммы 3.5 выполняются, и в силу указанной леммы найдутся случайные величины $Y_{j}^{*}, j \in J_{*}$, с функциями распределения из класса $\mathfrak{F}_{3, L^{2 c_{1}}}$, такие, что для них справедливы соотношения (3.6)-(3.8) с указанными выше значениями параметров $\eta$ и $K$. Из (3.6) получаем соотношение

$$
\left(1-L^{6 c_{1}}\right) \frac{1}{h^{2}} \sum_{j \in J_{*}} \sigma_{j}^{2} \leqslant \sum_{j \in J_{*}} \mathbf{D} Y_{j}^{*} \leqslant\left(1+L^{6 c_{1}}\right) \frac{1}{h^{2}} \sum_{j \in J_{*}} \sigma_{j}^{2},
$$

из которого, в силу $(2.10)$, следует, что

$$
\left(1-2 L^{6 c_{1}}\right) \frac{s^{2}}{h^{2}} \leqslant \sum_{j \in J_{*}} \mathrm{D} Y_{j}^{*} \leqslant\left(1+L^{6 c_{1}}\right) \frac{s^{2}}{h^{2}} .
$$

Кроме того, из (3.7) и (3.8) следует, что

$$
\sum_{j \in J_{*}}\left|\int_{|x| \leqslant L^{-5 / 12}} x^{3} d G_{j}(x)\right| \leqslant \sum_{j \in J_{*}}\left(\left|\mathbf{E}\left(Y_{j}^{*}-\mathbf{E} Y_{j}^{*}\right)^{3}\right|+L^{2 c_{1}} \mathbf{D} Y_{j}\right)
$$

и

$$
\sum_{j \in J_{*}} \mathbf{E}\left|Y_{j}^{*}-\mathbf{E} Y_{j}^{*}\right|^{3} \leqslant \sum_{j \in J_{*}}\left(\int_{|x| \leqslant L^{-5 / 12}}|x|^{3} d G_{j}(x)+L^{2 c_{1}} \mathbf{D} Y_{j}\right)
$$

соответственно.

Перепишем выражение для величины $\gamma_{J_{*}}$ в виде

$$
\begin{aligned}
\gamma_{J_{*}} & =\frac{1}{6 \sqrt{2 \pi}} \frac{h^{3}}{s^{3}} \sum_{j \in J_{*}}\left|\int_{|u| \leqslant \delta s / h} u^{3} d G_{j}(u)\right|+\frac{h}{2 \sqrt{2 \pi} s} \\
& =\frac{1}{6 \sqrt{2 \pi}} \frac{h^{3}}{s^{3}} \sum_{j \in J_{*}}\left|\int_{|u| \leqslant L-5 / 12} u^{3} d G_{j}(u)\right|+\frac{h}{2 \sqrt{2 \pi} s},
\end{aligned}
$$

и оценим ее с помощью (4.11) и (4.10). Получим

$$
\begin{aligned}
\gamma_{J_{*}} & \leqslant \frac{1}{6 \sqrt{2} \pi} \frac{h^{3}}{s^{3}}\left(\sum_{j \in J_{*}}\left|\mathbf{E}\left(Y_{j}^{*}-\mathbf{E} Y_{j}^{*}\right)^{3}\right|+L^{2 c_{1}} \frac{s^{2}}{h^{2}}\right)+\frac{h}{2 \sqrt{2 \pi} s} \\
& \leqslant \frac{1+L^{2 c_{1}}}{\sqrt{2 \pi}} \frac{h^{3}}{s^{3}} \sum_{j \in J_{*}}\left(\frac{1}{6}\left|\mathbf{E}\left(Y_{j}^{*}-\mathbf{E} Y_{j}^{*}\right)^{3}\right|+\frac{1}{2} \mathbf{D} Y_{j}^{*}\right) .
\end{aligned}
$$


Используя лемму 3.3 и неравенство (4.12), получаем

$$
\begin{aligned}
& \sum_{j \in J_{*}}\left(\frac{1}{6}\left|\mathbf{E}\left(Y_{j}^{*}-\mathbf{E} Y_{j}^{*}\right)^{3}\right|+\frac{\mathbf{D} Y_{j}^{*}}{2}\right) \leqslant \frac{\sqrt{10}+3}{6}\left(1+9 L^{2 c_{1}}\right) \sum_{j \in J_{*}} \mathbf{E}\left|Y_{j}^{*}-\mathbf{E} Y_{j}^{*}\right|^{3} \\
& \leqslant \frac{\sqrt{10}+3}{6}\left(1+9 L^{2 c_{1}}\right) \sum_{j \in J_{*}}\left(\int_{|x| \leqslant L^{-5 / 12}}|x|^{3} d G_{j}(x)+L^{2 c_{1}} \mathbf{D} Y_{j}\right)
\end{aligned}
$$

поэтому

$$
\begin{aligned}
\gamma_{J_{*}} & \leqslant\left(1+11 L^{2 c_{1}}\right) \frac{\sqrt{10}+3}{6 \sqrt{2 \pi}} \frac{h^{3}}{s^{3}} \sum_{j \in J_{*}}\left(\int_{|x| \leqslant L^{-5 / 12}}|x|^{3} d G_{j}(x)+L^{2 c_{1}} \mathbf{D} Y_{j}\right) \\
& \leqslant\left(1+11 L^{2 c_{1}}\right) \frac{\sqrt{10}+3}{6 \sqrt{2 \pi}} \frac{1}{s^{3}} \sum_{j \in J_{*}} \int_{|x| \leqslant \delta s}|x|^{3} d F_{j}(x)+c L^{2 c_{1}} \frac{h}{s} .
\end{aligned}
$$

Кроме того, $h / s \leqslant 10^{3} L$. Применяя последние два неравенства к (4.9), приходим к оценке

$$
\rho\left(F * \Phi_{\varepsilon}, \Phi\right) \leqslant \frac{\sqrt{10}+3}{6 \sqrt{2 \pi}} L+c L^{1+c_{1}}|\ln L|^{7 / 6} .
$$

С помощью леммы 3.1, так же, как и в доказательстве соотношения (4.4), получаем (2.14).

Докажем оценку (2.15). В силу (4.4), достаточно установить справедливость $(2.15)$ в случае, когда $M_{f}>L^{2}$ и $S_{*} \neq \varnothing$. Введем два подмножества $J_{*}^{(1)}$ и $J_{*}^{(2)}=J_{*} \backslash J_{*}^{(1)}$ множества $J_{*}$ следующим образом. Скажем, что индекс $j$ принадлежит $J_{*}^{(1)}$, если

$$
h \sigma_{j}^{2}>\frac{2}{3}\left|\int_{|u| \leqslant \delta s} u^{3} d F_{j}(u)\right|
$$

Пусть

$$
\zeta=-\frac{1}{6 s^{3}} \sum_{j=1}^{n} \int_{|u| \leqslant \delta s} u^{3} d F_{j}(u)
$$

Легко видеть, что

$$
\Phi(x)-\Phi(x-\zeta)=\zeta \Phi^{\prime}\left(x-\zeta_{1} \zeta\right)
$$

где $\zeta_{1} \in(0,1)$, и найдется $\zeta_{2} \in(0,1)$ такое, что

$$
\left|\Phi^{\prime}\left(x-\zeta_{1} \zeta\right)-\Phi^{\prime}(x)\right| \leqslant \frac{1}{\sqrt{2 \pi}} e^{-\left(x-\zeta_{2} \zeta\right)^{2} / 2}\left|x-\zeta_{2} \zeta\right||\zeta| .
$$

Поскольку $|\zeta| \leqslant L$, правая часть последнего неравенства не превосходит $c L$, так что

$$
\Phi(x)-\Phi(x-\zeta)=\zeta \frac{1}{\sqrt{2 \pi}} e^{-x^{2} / 2}+c \Theta L^{2} .
$$

Напомним, что $|\Theta| \leqslant 1$. Из последней формулы и (4.6) следует, что

$$
\left|\Phi(x)-\Phi(x-\zeta)+\alpha_{1}(x)\right| \leqslant \frac{1}{6 \sqrt{2 \pi}} x^{2} e^{-x^{2} / 2} \frac{1}{s^{3}} \sum_{j=1}^{n}\left|\int_{|u| \leqslant \delta s} u^{3} d F_{j}(u)\right|+c L^{19 / 12} .
$$

Отсюда и из представления (2.8), используя (4.5) и (4.8), получаем, что

$$
\begin{aligned}
\left|\left(F * \Phi_{\varepsilon}\right)(x)-\Phi(x-\zeta)\right| \leqslant \gamma_{J_{*}^{(1)}}(x)+\gamma_{J_{*}^{(2)}}+\frac{h}{2 \sqrt{2 \pi} s} m\left(\bar{J}_{*}\right) \\
\quad+\frac{1}{6 \sqrt{2 \pi} s^{3}} \sum_{j \in J_{*}} \int_{|u|>\delta s}|u|^{3} d F_{j}(u)+\frac{1}{6 \sqrt{2 \pi} s^{3}} \sum_{j \notin J_{*}} \beta_{j}+c L^{1+c_{1}}|\ln L|^{7 / 6}
\end{aligned}
$$


где

$$
\begin{aligned}
\gamma_{J_{*}^{(1)}}(x) & =\frac{1}{\sqrt{2 \pi}} e^{-x^{2} / 2} \frac{1}{s^{3}} \sum_{j \in J_{*}^{(1)}}\left(\frac{x^{2}}{6}\left|\int_{|u| \leqslant \delta s} u^{3} d F_{j}(u)\right|+\frac{h}{2} \sigma_{j}^{2}\right) \\
\gamma_{J_{*}^{(2)}} & =\frac{1}{\sqrt{2 \pi}} \frac{1}{s^{3}} \sum_{j \in J_{*}^{(2)}}\left(\frac{1}{6}\left|\int_{|u| \leqslant \delta s} u^{3} d F_{j}(u)\right|+\frac{h}{2} \sigma_{j}^{2}\right) .
\end{aligned}
$$

При условии (4.13),

$$
\sup _{x \in \mathbf{R}^{1}} e^{-x^{2} / 2}\left(\frac{x^{2}}{6}\left|\int_{|u| \leqslant \delta s} u^{3} d F_{j}(u)\right|+\frac{h}{2} \sigma_{j}^{2}\right) \leqslant \frac{h}{2} \sigma_{j}^{2} .
$$

Отсюда, в силу (3.6) при $X=Y_{j}$ и $X^{*}=Y_{j}^{*}, j \in J_{*}$, получаем

$$
\gamma_{J_{*}^{(1)}}(x) \leqslant \frac{1}{\sqrt{2 \pi}} \frac{1}{s^{3}} \sum_{j \in J_{*}^{(1)}} \frac{h}{2} \sigma_{j}^{2}=\frac{1}{\sqrt{2 \pi}} \frac{h^{3}}{s^{3}} \sum_{j \in J_{*}^{(1)}} \frac{1}{2} \mathbf{D} Y_{j} \leqslant \frac{1+L^{6 c_{1}}}{\sqrt{2 \pi}} \frac{h^{3}}{s^{3}} \sum_{j \in J_{*}^{(1)}} \frac{1}{2} \mathbf{D} Y_{j}^{*} .
$$

Согласно лемме 3.2 , для случайных величин $Y_{j}^{*}, j \in J_{*}$, верно соотношение

$$
\frac{1}{2}\left(1-8 L^{2 c_{1}}\right) \mathbf{D} Y_{j}^{*} \leqslant \mathbf{E}\left|Y_{j}^{*}-\mathbf{E} Y_{j}^{*}\right|^{3} .
$$

Используя (4.15) и (4.12), получаем, наконец,

$$
\begin{aligned}
\gamma_{J_{*}^{(1)}}(x) & \leqslant \frac{1+L^{c_{1}}}{\sqrt{2 \pi}} \frac{h^{3}}{s^{3}} \sum_{j \in J_{*}^{(1)}} \mathbf{E}\left|Y_{j}^{*}-\mathbf{E} Y_{j}^{*}\right|^{3} \\
& \leqslant \frac{1+L^{c_{1}}}{\sqrt{2 \pi}} \frac{1}{s^{3}}\left(\sum_{j \in J_{*}^{(1)}} \int_{|u| \leqslant \delta s}\left|u^{3}\right| d F_{j}(u)+L^{2 c_{1}} h s^{2}\right) .
\end{aligned}
$$

Согласно определению множества $J_{*}^{(2)}$, величина $\gamma_{J_{*}^{(2)}}$ не превосходит

$$
\frac{1}{\sqrt{2 \pi}} \frac{1}{s^{3}} \sum_{j \in J_{*}^{(2)}} \int_{|u| \leqslant \delta s}\left|u^{3}\right| d F_{j}(u) .
$$

Применяя (4.16) и (4.17) к (4.14) и учитывая (2.9) и (2.10), получаем неравенство

$$
\left|\left(F * \Phi_{\varepsilon}\right)(x)-\Phi(x-\zeta)\right| \leqslant \frac{1}{\sqrt{2 \pi}} L+c L^{1+c_{1}}|\ln L|^{7 / 6} .
$$

Легко видеть, что из этого неравенства следует соотношение

$$
\left|\left(F * \Phi_{\varepsilon}\right)(x)-\left(\Phi * \Phi_{\varepsilon}\right)(x-\zeta)\right| \leqslant \frac{1}{\sqrt{2 \pi}} L+c L^{1+c_{1}}|\ln L|^{7 / 6} .
$$

Для доказательства (2.15) остается применить лемму 3.1 к последнему неравенству, как и в доказательстве (4.4).

Д ок а з а те ль с т в о о е н к и (2.16). Будем рассуждать так же, как и при доказательстве (2.14) и используем те же обозначения. В силу (4.4), достаточно установить справедливость $(2.16)$ в случае, когда $M_{f}>L^{2}$ и $S_{*} \neq \varnothing$. Используем вновь соотношение (4.9), в котором выражение для $\gamma_{J_{*}}$ имеет вид $\gamma_{J_{*}}=h /(2 \sqrt{2 \pi} s)$ и тогда, в силу (2.10) и (3.6), при $X=Y_{j}, X^{*}=Y_{j}^{*}, j \cdot \in J_{*}$, справедлива оценка

$$
\gamma_{J_{*}} \leqslant \frac{1+L^{7 c_{1}}}{\sqrt{2 \pi}} \frac{1}{s^{3}} \sum_{j \in J_{*}} \frac{h}{2} \sigma_{j}^{2} \leqslant \frac{1+L^{6 c_{1}}}{\sqrt{2 \pi}} \frac{h^{3}}{s^{3}} \sum_{j \in J_{*}} \frac{1}{2} \mathrm{D} Y_{j}^{*} .
$$


Из этого неравенства и оценок (4.15) и (4.12) следует, что

$$
\begin{aligned}
\gamma_{J_{*}} & \leqslant \frac{1+L^{c_{1}}}{\sqrt{2 \pi}} \frac{h^{3}}{s^{3}} \sum_{j \in J_{*}} \mathbf{E}\left|Y_{j}^{*}-\mathbf{E} Y_{j}^{*}\right|^{3} \\
& \leqslant \frac{1+L^{c_{1}}}{\sqrt{2 \pi}} \frac{1}{s^{3}} \sum_{j \in J_{*}}\left(\int_{|u| \leqslant \delta s}|u|^{3} d F_{j}(u)+L^{2 c_{1}} h \sigma_{j}^{2}\right) \\
& =\frac{1+L^{c_{1}}}{\sqrt{2 \pi}}\left(\frac{1}{s^{3}} \sum_{j \in J_{*}} \int_{|u| \leqslant \delta s}|u|^{3} d F_{j}(u)+L^{2 c_{1}} \frac{h}{s}\right) .
\end{aligned}
$$

Применяя эту оценку к (4.9) и учитывая, что $h / s \leqslant 10^{3} L$, легко получаем, что

$$
\rho\left(F * \Phi_{\epsilon}, \Phi\right) \leqslant \frac{1}{\sqrt{2 \pi}} L+c L^{1+c_{1}}|\ln L|^{7 / 6} .
$$

Теперь неравенство (2.16) следует из леммы 3.1. Равенство $c_{s}=1 / \sqrt{2 \pi}$ вытекает из этого неравенства и второй части теоремы В.

Остается заметить, что следствие 2.3 непосредственно следует из теоремы 2.2 , если учесть вторые части теорем А и $\mathrm{B}$.

Часть настоящих исследований бьла осуществлена в Университете Билефельда. Я благодарен проф. Ф. Гётце за его гостеприимство во время моего пребывания в Билефельде. Я также благодарен В. Бенткусу, Ф. Гётце и А. Ю. Зайцеву за полезные обсуждения.

\section{СПИСОК ЛИТЕРАТУРЫ}

1. Араж T.В., Зайщеє А. Ю. Равномерные предельные теоремы для сумм независимых случайных величин. - Труды Матем. ин-та им. В. А. Стеклова, 1986, T. 174.

2. Bentkus $V$. On the asymptotical behavior of the constant in the Berry-Esseen inequality. - J. Theor. Probab., 1994, v. 7, № 2, p. 211-224.

3. Berry A.C. The accuracy of the Gaussian approximation to the sum of independent variates. - Trans. Amer. Math. Soc., 1941, v. 49, p. 122-136.

4. Чистлков Г. П. Об одной задаче Колмогорова. - Записки научн. семин. ЛОМИ, 1990, т. 184, c. $289-319$.

5. Esseen C.-G. On the Liapounoff limit of error in the theory of probability. - Ark. Mat. Astron. Fys., 1942, v. A28, № 9, p. 1-19.

6. Esseen C.-G. Fourier analysis of distribution functions. A mathematical study of the Laplace-Gaussian law. - Acta Math., 1945, v. 77, p. 1-125.

7. Esseen C.-G. A moment inequality with an application to the central limit theorem. Skand. Aktuarietidskr., 1956, v. 39, p. 160-170.

8. Ибрагимов И.А., Линник ЮО.В. Независимые и стационарно связанные величины. М.: Наука, 1965, 524 с.

9. Колмогоров А. Н. Некоторые работы последних лет в области предельных теорем теории вероятностей. - Вестник Московского унив., 1953, № 10, с. 29-38.

10. Le Cam $L$. A note on the distribution of sums of independent random variables. Proc. Natl. Acad. Sci. USA, 1963, v. 50, p. 601-603.

11. Линник ЮO. $B$. О точности приближения к гауссову распределению сумм независимых случайных величин. - Изв. АН СССР, сер. матем., 1947, т. 11, № 2, c. $111-138$.

12. Петрое B. B., О точных оценках в предельных теоремах. - Докл. АН СССР, 1955, т. 104 , № 2, c. 180-182.

13. Петров B. B., Суммы независимых случайных величин. М.: Наука, 1972, 416 с.

14. Петров B. B., Предельные теоремы для сумм независимых случайных величин. М.: Наука, 1987.

15. Рогозия B. A. Одно замечание к работе Эссеена «Моментное неравенство с прйменением к центральной предельной теореме». - Теория вероятн. и ее примен., 1960 , т. 5, в. 1, C. $125-128$. 
16. Золотарев B. M. Современная теория суммирования независимых случайных величин. М.: Наука, 1986.

Поступила в редакцию 30.VI.1998

(C) $2001 \Gamma$.

KALLSEN J. ${ }^{*}$, SHIRYAEV A. N.**

\section{TIME CHANGE REPRESENTATION OF STOCHASTIC INTEGRALS ${ }^{1)}$}

По теореме Дамбиса и Дубинса-Шварца любой стохастический интеграл $M=\left(\int_{0}^{t} H_{s} d W_{s}\right)_{t \in \mathbf{R}_{+}}$по броуновскому движению может быть записан как броуновское движение со случайной заменой времени, т.е. $M=\left(\widehat{W}_{\widehat{T}_{t}}\right)_{t \in \mathbf{R}_{+}}$для некоторого броуновского движения $\left(\widehat{W}_{\theta}\right)_{\theta \in \mathbf{R}_{+}}$и некоторой замены времени $\left(\widehat{T}_{t}\right)_{t \in \mathbf{R}_{+}}$. В [7] и [5] показано, что в этом утверждении броуновское движение можно заменить на (симметричное) $\alpha$-устойчивое дөижение Леви. Используя процесс кумулянт семимартингала, мы даем короткие новые доказательства. Кроме того, мы показываем, что это утверждение не может быть распространено на другие процессы Леви.

Ключевые слова и фразы: устойчивые движения Леви, кумулянтный процесс, стохастический интеграл, замена времени.

1. Time change representations. We generally use the notation of [2]-[4]. The transposed of a vector or matrix $x$ is denoted as $x^{\top}$ and its components are denoted by superscripts. Stochastic and Stieltjes integrals are written as $\left(H \cdot X_{t}\right)=\int_{0}^{t} H_{s} d X_{s}$. Increasing processes are identified with their corresponding Lebesgue-Stieltjes measure.

Let $\left(\Omega, \mathscr{F},\left(\mathscr{F}_{t}\right)_{t \in \mathbf{R}_{+}}, \mathbf{P}\right)$ be a filtered probability space as in [4, Definition I.1.2]. By Lévy process we refer to a process with stationary independent increments in the sense of $[4$, II.4.1]. Similar to [8, Definition 7.5.1], we make the following

$\mathrm{D}$ e f i $\mathrm{n}$ i t i o $\mathrm{n} 1$. Let $\alpha \in(0,2]$. An $\alpha$-stable Lévy motion is a Lévy process $X$ such that $X_{1}$ (or equivalently any $X_{t}$ ) has a strictly $\alpha$-stable distribution (i.e., $X_{1} \sim$ $S_{\alpha}(\sigma, \beta, \mu)$ for some $\alpha \in(0,2] \backslash\{1\}, \sigma \in \mathbf{R}_{+}, \beta \in[-1,1], \mu=0$ or $\alpha=1, \sigma \in \mathbf{R}_{+}$, $\beta=0, \mu \in \mathbf{R}$ ). We call $X$ a symmetric $\alpha$-stable Lévy motion if the distribution of $X_{1}$ (or equivalently any $\left.X_{t}\right)$ is even symmetric $\alpha$-stable (i.e., $X_{1} \sim S_{\alpha}(\sigma, 0,0)$ for some $\alpha \in(0,2]$, $\sigma \in \mathbf{R}_{+}$).

$\mathrm{D}$ e f $\mathrm{i} \mathrm{n}$ i $\mathrm{t}$ i o $\mathrm{n}$ 2. 1. A time change is a right-continuous increasing $[0, \infty]$-valued process $\left(T_{\theta}\right)_{\theta \in \mathbf{R}_{+}}$such that $T_{\theta}$ is a stopping time for any $\theta \in \mathbf{R}_{+}$. It is called finite if $T_{\theta}<\infty \mathbf{P}$-almost surely for any $\theta \in \mathbf{R}_{+}$.

2. By $\widehat{\mathscr{F}}_{\theta}:=\mathscr{F}_{T_{\theta}}$ we define the time-changed filtration $\left(\widehat{\mathscr{F}}_{\theta}\right)_{\theta \in \mathbf{R}_{+}}$.

3. The inverse time change $\left(\widehat{T}_{t}\right)_{t \in \mathbf{R}_{+}}$is defined as $\widehat{T}_{t}:=\inf \left\{\theta \in \mathbf{R}_{+}: T_{\theta}>t\right\}$.

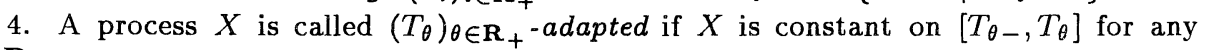
$\theta \in \mathbf{R}_{+}$.

R. e m a r k. If $X$ is a semimartingale on $\left(\Omega, \mathscr{F},\left(\mathscr{F}_{t}\right)_{t \in \mathbf{R}_{+}}, \mathbf{P}\right)$ and $\left(T_{\theta}\right)_{\theta \in \mathbf{R}_{+}}$is a finite time change, then the process $\left(\widehat{X}_{\theta}\right)_{\theta \in \mathbf{R}_{+}}$defined by $\widehat{X}_{\theta}:=X_{T_{\theta}}$ is a semimartingale

* Universität Freiburg, Institut für Mathematische Stochastik, Eckerstr., 1, D79104 Freiburg, Germany; e-mail: kallsen@neyman.mathematik.uni-freiburg.de

** Математический институт им. В. А. Стеклова РАН, ул. Губкина, 8, 117966 Москва, ГСП-1, Россия; e-mail: shiryaev@mi.ras.ru

1) Работа второго автора поддержана Alexander von Humboldt Foundation и Российским фондом фундаментальных исследований (грант № 99-01-00152). 